\title{
A Nuclear Ring in the Sa(r!) Galaxy NGC 7742
}

\author{
K. Wakamatsu ${ }^{1}$ \\ Physics Department, Gifu University, Gifu 501-11, Japan \\ M. Hamabe
}

Institute of Astronomy, The University of Tokyo, Mitaka 181, Japan

M. T. Nishida ${ }^{1}$

Kobe Women's University, Kobe 652, Japan

\author{
A. Tomita \\ Department of Astronomy, Kyoto University, Kyoto 606, Japan
}

\section{Introduction}

NGC 7742 is well known for its prominent blue nuclear ring around an E0-like core, and so appears as a Hoag-type galaxy, an elliptical galaxy with an outer ring (Schweizer et al. 1987). The galaxy is classified as $\mathrm{Sa}(\mathrm{r} !)$ in the Revised Shapley-Ames Catalog (Sandage and Tammann 1987) with an exclamation mark to emphasize the prominence of the ring. Its photographs are published in Laustsen et al. (1987), Wray (1988), and Sandage \& Bedke (1994).

The ring has a diameter of $19^{\prime \prime}=1.6 \mathrm{kpc}$ at a distance of $17.1 \mathrm{Mpc}$ (Buta $\&$ Crocker 1993), and so should be a nuclear ring of the galaxy. Nuclear rings and pseudorings are often detected in strongly barred (SB) galaxies, and interpreted to be linked to the inner Lindblad resonance (Buta \& Crocker 1993). These nuclear features are, however, also found in some weakly-barred (SAB) and nonbarred (SA) galaxies. NGC 7742 is a galaxy of the highest circular symmetry in its core, ring, and main body, and so the best object for a detailed study of formation mechanisms of nuclear rings in non-barred galaxies.

\section{Observations and Results}

In order to study the kinematics of the ring, spectra in the $\mathrm{H} \alpha$ region with a dispersion of $1.5 \AA$ pix $^{-1}$ were taken with the Okayama 1.9 -m Cassegrain spectrograph at 6 different position angles at an interval of $30^{\circ}$. For a study of star forming activity and metallicity gradients, flux calibrated spectra in a wavelength region of $3400 \AA<\lambda<7500 \AA$ were kindly secured by Matt Malkan with

\footnotetext{
${ }^{1}$ Visiting Astronomer, Cerro Tololo Inter-American Observatory. CTIO is operated by AURA, Inc. under cooperative agreement with the National Science Foundation
} 


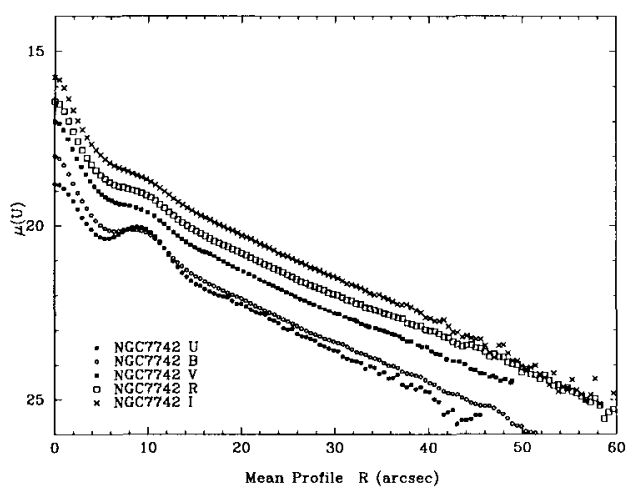

Figure 1. Radial luminosity profiles of NGC 7742

the Lick 3-m KAST spectrograph. CCD images were taken with the CTIO 0.9$\mathrm{m}$ telescope in the standard $U, B, V, R$, and $I$ bands with a scale of $0.49 \mathrm{pix}^{-1}$. A $V$-band image of HST archival data was also used.

On the HST image, the nuclear ring is clearly resolved into tightly wound flocculent spiral arms with many compact HII regions and star clusters. The arms wind clockwise, and are delineated by dust lanes in their inner edges. The central core has several faint dust lanes at its periphery. Therefore, the core plus ring of NGC 7742 looks like a mini face-on Sb galaxy embedded in the main body of the galaxy as is the case in the nuclear spiral pattern in NGC 4314 (Benedict et al 1993). Outside the ring, very faint flocculent spiral arms can be seen on $U$ and $B$ CCD frames as shown in an $\mathrm{H} \alpha$ image (Pogge \& Eskridge 1993). They wind clockwise and less tightly than the nuclear ring.

Azimuthally-averaged radial luminosity profiles are shown in Figure 1. The nuclear ring is identified as bumps at $r=9-11^{\prime \prime}$. It is most prominent in $U$-band, but fades away as redder images are concerned, though still visible in $I$-band. An exponential disk is well defined beyond $r=13^{\prime \prime}$. The profiles are typical of Freeman's type I profile with parameters $B(0)_{c}=19.7 \mathrm{mag} \mathrm{arcsec}{ }^{-2}$ and $\alpha^{-1}=10^{\prime \prime}=0.83 \mathrm{kpc}$. The existence of the exponential disk definitely rules out that NGC 7742 is a Hoag-type galaxy.

On our isophotal maps of CTIO images, both the ring and the exponential disk can be fitted with similar ellipses of $b / a=0.97 \pm 0.05$ with the major axis at $\mathrm{PA}=25^{\circ}$. The galaxy rotates as a solid body up to the ring radius, and in a flat rotation curve beyond the ring. Rotational velocities measured at the ring can be fitted to a sinusoidal curve as a function of the position angle, and the line of nodes lies at $\mathrm{PA}=38^{\circ} \pm 10^{\circ}$. The maximum rotational velocity of the galaxy after inclination correction is estimated to be $145 \pm 15 \mathrm{~km} \mathrm{~s}^{-1}$. This is a reasonable value for its absolute blue magnitude of $M_{T}^{\circ}=-18.9$.

Though undetected on the CTIO data at a level of a few percent, an oval structure is definitely detected on the HST data at the periphery of the central core $\left(r \sim 5^{\prime \prime}\right)$ inside the ring. As shown in Figure 2, isocontours are elongated both at $r=5^{\prime \prime} .1$ and $1^{\prime \prime} 5$ with a twist of the major axes from PA $=10^{\circ} \pm 10^{\circ}$ to $110^{\circ} \pm 10^{\circ}$. The axial ratio is $b / a=0.86 \pm 0.05$ at $r \sim 5^{\prime \prime}$. In order to examine quantitatively whether the oval components are strong enough to form 

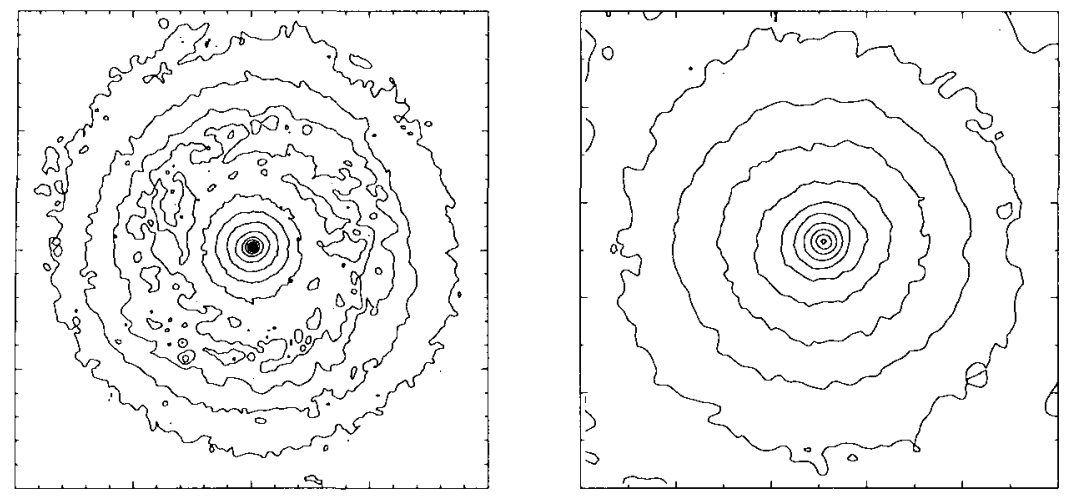

Figure 2. Isophotes of NGC 7742 (left: $40^{\prime \prime} \times 40^{\prime \prime}$, right: $10^{\prime \prime} \times 10^{\prime \prime}$ )

the nuclear ring, we extracted azimuthal luminosity profiles along circles on the disk plane at various radii. They are to be analyzed with Fourier decomposition as we did for barred galaxies (Ohta et al. 1990). Results of a radial change of Fourier components of $m=2$ and 4 will be given in a forthcoming paper.

\section{References}

Benedict, G. F. et al. 1993, AJ, 105, 1369

Buta, R. \& Crocker, D. A. 1993, AJ, 105, 1344

Laustsen, S. et al. 1987, Exploring the Southern Sky, New York: Springer-Verlag Ohta, K., Hamabe, M., \& Wakamatsu, K. 1990, ApJ, 357, 71

Pogge, R. W. \& Eskridge, P. B. 1993, AJ, 106, 1405

Sandage, A. \& Bedke, J. 1994, The Carnegie Atlas of Galaxies, Carnegie Institution of Washington Publ. 638

Sandage, A. \& Tammann, G. 1987, A Revised Shapley-Ames Catalog, Carnegie Institution of Washington Publication No. 635

Schweizer, F. et al. 1987, ApJ, 320, 454

Wray, J. D. 1988, The Color Atlas of Galaxies, Cambridge: Cambridge Univ. Press

\section{Discussion}

B. Elmegreen: One possible model for this ring without a bar is that it arises from viscous gas accretion to a stagnation point at the turnover radius of the rotation curve.

J. Palous: The ring may be formed due to molecular cloud collisions in an almost axisymmetric potential of the galaxy perturbed by a weak $(<1 \%)$ bar or triaxial bulge/halo (see the paper by Palouš in these proceedings). 